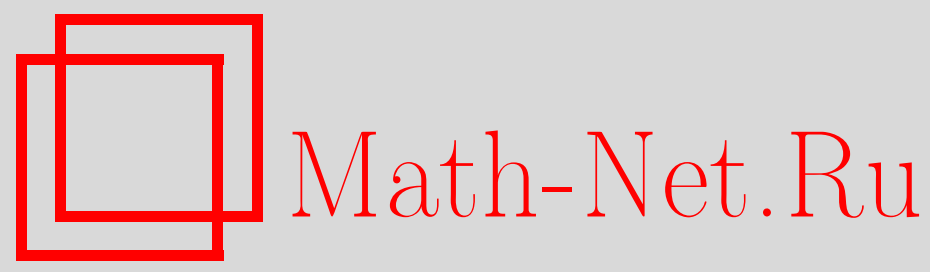

Б. В. Чокаев, Сложность умножения в коммутативных групповых алгебрах над полями простой характеристики, Дискрет. матем., 2010, том 22, выпуск 4, 121-137

DOI: https://doi.org/10.4213/dm1123

Использование Общероссийского математического портала Math-Net.Ru подразумевает, что вы прочитали и согласны с пользовательским соглашением http://www.mathnet.ru/rus/agreement

Параметры загрузки:

IP : 34.239 .49 .27

26 апреля 2023 г., 13:33:14 
УДК 519.7

\title{
Сложность умножения в коммутативных групповых алгебрах над полями простой характеристики
}

\author{
(c) 2010 г. $\quad$ Б. В. Чокаев
}

\begin{abstract}
Целью данной работы является исследование сложности умножения в коммутативных групповых алгебрах над произвольными полями простой характеристики. Для решения этой задачи предложен метод нахождения структуры групповых алгебр, который позволяет использовать теорему Алдера-Штрассена для получения нижних оценок и теорему Блезера, описывающую все алгебры минимального ранга, для получения верхних оценок.
\end{abstract}

\section{1. Введение}

Одной из наиболее важных и интересных задач в алгебраической теории сложности является задача сложности умножения матриц. В 2003 г. Г. Коэн и К. Уманс предложили новый подход для получения верхних оценок сложности умножения матриц, основанный на вложениях в групповые алгебры (см. [1]). В 2005 г. были получены первые алгоритмы умножения матриц с оценкой сложности ниже, чем $O\left(n^{3}\right)$ (см. [2]). Несмотря на то, что все улучшения, полученные этим подходом, по сути представляют собой изложение классических результатов, переписанное на теоретико-групповом языке, это стимулировало рост интереса к групповым алгебрам.

В кандидатской диссертации А. Д. Поспелова (см. [3]) были рассмотрены коммутативные групповые алгебры над алгебраически замкнутыми полями и полем вещественных чисел. Им были установлены точные значения билинейной сложности умножения в таких групповых алгебрах. Этот результат является интересным потому, что любая нижняя оценка в алгебре над алгебраически замкнутым полем является универсальной, то есть она верна в алгебре над произвольным полем, алгебраическое замыкание которого совпадает с данным полем.

Целью данной работы является исследование сложности умножения в коммутативных групповых алгебрах над произвольными полями простой характеристики. Для решения этой задачи предложен метод нахождения структуры групповых алгебр, который позволяет использовать теорему Алдера-Штрассена для получения нижних оценок и теорему Блезера, описывающую все алгебры минимального ранга, для получения верхних оценок.

\section{1. Основные понятия}

Алгеброй над полем $k$ называется конечномерное линейное пространство $A$ над $k$, в котором определено умножение пар векторов, линейное по обоим аргументам: для $a$ и $b$ 
из $A$ определен вектор $c=a b$ также из $A$, причем

$$
(\alpha a) b=a(\alpha b)=\alpha(a b)
$$

для любого $\alpha$ из $k$ и

$$
(a+d) b=a b+d b, \quad a(b+d)=a b+a d
$$

для любого $d$ из $A$. При этом размерность соответствующего линейного пространства называется также размерностью алгебры и обозначается $\operatorname{dim} A$, а базис линейного пространства называется базисом алгебры.

Пусть $A$ и $B$ - алгебры над $k$. Прямым произведением $A$ и $B$ называется алгебра $C=A \times B$ над $k$ размерности $\operatorname{dim} C=\operatorname{dim} A+\operatorname{dim} B$, элементы которой являются парами элементов $(a, b)$, где $a \in A, b \in B$. Сложение и умножение в $C$ производится покомпонентно:

$$
\begin{aligned}
\alpha(a, b)+\beta(c, d) & =(\alpha a+\beta c, \alpha b+\beta d), \\
(a, b)(c, d) & =(a c, b d) .
\end{aligned}
$$

Алгебра называется неразложимой, если ее нельзя представить в виде прямого произведения двух алгебр. Произвольная разложимая алгебра может быть представлена в виде прямого произведения неразложимых алгебр, причем это представление единственно с точностью до перестановки сомножителей (см. [4]).

Пусть $A$ и $B$ - алгебры над полем $k$, и $\left\{a_{1}, \ldots, a_{m}\right\},\left\{b_{1}, \ldots, b_{n}\right\}-$ соответственно базисы $A$ и $B$, умножение в которых определяется следующим образом:

$$
\begin{array}{ll}
a_{i} a_{j}=\sum_{\mu=1}^{m} \alpha_{\mu}^{i j} a_{\mu}, & 1 \leqslant i, j \leqslant m, \\
b_{r} b_{s}=\sum_{\nu=1}^{n} \beta_{\nu}^{r s} b_{\nu}, & 1 \leqslant r, s \leqslant n .
\end{array}
$$

Определим тензорное произведение $A$ и $B: C=A \otimes B$ над $k$ размерности $m n$ с базисом $\left\{c_{(1,1)}, \ldots, c_{(m, n)}\right\}$ и правилом умножения

$$
c_{(i, r)} c_{(j, s)}=\sum_{\mu=1}^{m} \sum_{\nu=1}^{n} \alpha_{\mu}^{i j} \beta_{\nu}^{r s} c_{(\mu, \nu)}, \quad 1 \leqslant i, j \leqslant m, \quad 1 \leqslant r, s \leqslant n .
$$

Легко видеть, что для произвольной алгебры $A$ над полем $k$ выполняется соотношение

$$
A \otimes k \cong A \text {. }
$$

Также можно доказать, что обе операции взятия прямого и тензорного произведений алгебр являются коммутативными и ассоциативными, и для произвольных алгебр $A, B$, $C$, определенных над одним и тем же полем, выполняется свойство дистрибутивности:

$$
(A \times B) \otimes C \cong(A \otimes C) \times(B \otimes C) .
$$

Пусть $A$ - алгебра над полем $k$ размерности $n$, и пусть $a_{1}, \ldots, a_{n}-$ некоторый базис $A$. Если множество $\left\{a_{1}, \ldots, a_{n}\right\}$ образует группу относительно умножения в $A$, то такой 
базис называется групповым базисом, а алгебра, соответственно, групповой алгеброй. Обратно, пусть $G=\left\{g_{1}, \ldots, g_{n}\right\}$ - группа порядка $n$, и $k-$ поле. Тогда алгебра

$$
B=\left\{\alpha_{1} g_{1}+\ldots+\alpha_{n} g_{n} \mid \alpha_{v} \in k, 1 \leqslant v \leqslant n\right\}
$$

с умножением, определяемым по правилу

$$
\left(\sum_{\nu=1}^{n} \alpha_{\nu} g_{\nu}\right)\left(\sum_{\mu=1}^{n} \beta_{\mu} g_{\mu}\right)=\sum_{\kappa=1}^{n}\left(\sum_{\nu, \mu: g_{\nu} g_{\mu}=g_{\kappa}} \alpha_{\nu} \beta_{\mu}\right) g_{\kappa},
$$

является групповой. Будем обозначать алгебру над $k$ с групповым базисом, образующим группу $G$, через $k[G]$.

Пусть $A$ и $B-$ групповые алгебры над полем $k$, и

$$
\begin{aligned}
G_{A} & =\left\{a_{1}, \ldots, a_{m}\right\}, \\
G_{B} & =\left\{b_{1}, \ldots, b_{n}\right\}
\end{aligned}
$$

- соответственно их групповые базисы, причем

$$
\begin{array}{ll}
a_{i} a_{j}=a_{\varphi(i, j)}, & 1 \leqslant i, j \leqslant m, \\
b_{r} b_{s}=b_{\psi(r, s)}, & 1 \leqslant r, s \leqslant n .
\end{array}
$$

Тогда одним из базисов алгебры $C=A \otimes B$ будет множество $\left\{c_{(1,1)}, \ldots, c_{(m, n)}\right\}$, умножение в котором определяется правилом

$$
c_{(i, r)} c_{(j, s)}=c_{(\varphi(i, j), \psi(r, s)),} \quad 1 \leqslant i, j \leqslant m, \quad 1 \leqslant r, s \leqslant n .
$$

Заметим, что правая часть, согласно определению, соответствует умножению в $k\left[G_{A} \times G_{B}\right]$. Таким образом,

$$
k\left[G_{A}\right] \otimes k\left[G_{B}\right] \cong k\left[G_{A} \times G_{B}\right] .
$$

\section{2. Модель вычислений}

Для того, чтобы решать задачу сложности умножения в алгебре, необходимо зафиксировать модель вычислений и функционал сложности. Общепринятой в алгебраической теории сложности является билинейная модель вычисления (см. [5]). Билинейным вычислением (билинейным алгоритмом) для алгебры $A$ называется такая последовательность $\left(f_{1}, g_{1}, w_{1}, \ldots, f_{r}, g_{r}, w_{r}\right)$, где $f_{\rho}, g_{\rho} \in A^{*}, w_{\rho} \in A, 1 \leqslant \rho \leqslant r$, что для любых $a, b \in A$

$$
a \cdot b=\sum_{\rho=1}^{r} f_{\rho}(a) g_{\rho}(b) w_{\rho}
$$

Число $r$ называется длиной билинейного алгоритма. Длина кратчайшего билинейного алгоритма для $A$ называется рангом (билинейной сложностью) и обозначается $\operatorname{rk} A$.

Пусть $A$ и $B$ - произвольные алгебры над одним и тем же полем. Тогда справедливы следующие соотношения (см. [6]):

$$
\begin{aligned}
& \operatorname{rk}(A \times B) \leqslant \operatorname{rk} A+\operatorname{rk} B, \\
& \operatorname{rk}(A \otimes B) \leqslant \operatorname{rk} A \cdot \operatorname{rk} B .
\end{aligned}
$$

Существует гипотеза (гипотеза о прямой сумме) о том, что неравенство в (4) выполняется как равенство для произвольных алгебр $A$ и $B$ (см. [6]). 


\section{3. Используемые методы}

Подалгебра $I$ алгебры $A$ называется (двусторонним) идеалом $A$, если для любых $a \in A$, $b \in I$ справедливы включения $a b \in I, b a \in I$. Идеал $I \neq A$ называется максимальным, если из $I \subsetneq J$, где $J-$ идеал в $A$, следует, что $J=A$. Одним из фундаментальных результатов о билинейной сложности алгебр является теорема Алдера-Штрассена.

Теорема 1 ([7]). Для произвольной ассочиативной алгебры А выполняется неравенство

$$
\text { rk } A \geqslant 2 \operatorname{dim} A-t(A),
$$

где $t(A)$ - число максимальных двусторонних идеалов А. Алгебры, для которых

$$
\text { rk } A=2 \operatorname{dim} A-t(A),
$$

называются алгебрами минимального ранга.

Прежде чем дать описание структуры алгебры минимального ранга над произвольным полем, дадим несколько определений. Идеал $I$ в $A$ называется нильпотентным, если для некоторого целого числа $n$ произведение любых $n$ элементов идеала $I$ равно нулю. Объединение всех нильпотентных идеалов алгебры $A$ является нильпотентным идеалом в $A$, называется радикалом $A$ и обозначается $\operatorname{rad} A$. Известно (см. [8]), что $\operatorname{rad} A$ содержится в любом максимальном идеале $A$. Будем называть алгебру $A$ над полем $k$ сверхосновной, если фактор-алгебра $A / \operatorname{rad} A$ изоморфна алгебре $k \times k \times \ldots \times k$.

Теорема 2 ([9]). Алгебра А над полем $k$ является алгеброй минимального ранга тогда $u$ только тогда, когда

$$
A \cong C_{1} \times \ldots \times C_{S} \times k^{2 \times 2} \times \ldots \times k^{2 \times 2} \times B_{1} \times \ldots \times B_{r},
$$

где $C_{1}, \ldots, C_{s}$ суть локальные алгебры минимального ранга, у которых

$$
\operatorname{dim}\left(C_{\sigma} / \operatorname{rad} C_{\sigma}\right) \geqslant 2
$$

mo ecmb

$$
C_{\sigma} \cong k[X] /\left(p_{\sigma}(X)^{d_{\sigma}}\right)
$$

для некоторого неприводимого над $k$ полинома $p_{\sigma}, \operatorname{deg} p_{\sigma} \geqslant 2, d_{\sigma} \geqslant 1,|k| \geqslant 2 \operatorname{dim} C_{\sigma}-2$, $\sigma=1, \ldots, s, k^{2 \times 2} \times \ldots \times k^{2 \times 2}-$ произведение и множителей, а $B_{1}, \ldots, B_{r}-$ неразложимые сверхосновные алгебры минимального ранга над $k$. Сверхосновная алгебра $B$ является алгеброй минимального ранга тогда и только тогда, когда найдутся такие $w_{1}, \ldots, w_{m} \in \operatorname{rad} B, w_{i} w_{j}=0, i \neq j$, что

$$
\operatorname{rad} B=L_{B}+B w_{1} B+\ldots+B w_{m} B=R_{B}+B w_{1} B+\ldots+B w_{m} B
$$

u $|k| \geqslant 2 N(B)-2$, где $L_{B}$ и $R_{B}$ суть правый и левый аннигиляторы $\operatorname{rad} B$ (то есть множество всех таких $x \in \operatorname{rad} B$, что $x(\operatorname{rad} B)=0$, соответственно $(\operatorname{rad} B) x=0), a$ $N(B)$ - наибольшее цчелое $j$, для которого $(\operatorname{rad} B)^{j} \neq\{0\}$. Любое из чисел $s, u, r$ может равняться нулю.

Рассмотрим алгебру $k\left[X_{1}, \ldots, X_{m}\right]$ многочленов над полем $k$ от переменных $X_{1}, \ldots, X_{m}$. Пусть

$$
A \cong k\left[X_{1}, \ldots, X_{m}\right] /\left(X_{1}^{n_{1}}-1, \ldots, X_{m}^{n_{m}}-1\right)
$$


- фактор-алгебра алгебры $k\left[X_{1}, \ldots, X_{m}\right]$ по идеалу, порожденному многочленами $X_{1}^{n_{1}}-1, \ldots, X_{m}^{n_{m}}-1$. Алгебра $A$ изоморфна алгебре $k[G]$ при

$$
G \cong \mathbf{Z}_{n_{1}} \times \mathbf{Z}_{n_{2}} \times \ldots \times \mathbf{Z}_{n_{m}} .
$$

Действительно, обозначим элементы $G$ через $g_{i_{1}, \ldots, i_{m}}, 0 \leqslant i_{\mu}<n_{\mu}$, причем

$$
g_{i_{1}, \ldots, i_{m}} g_{j_{1}, \ldots, j_{m}}=g_{\left(i_{1}+j_{1}\right)}\left(\bmod n_{1}\right), \ldots,\left(i_{m}+j_{m}\right) \quad\left(\bmod n_{m}\right)
$$

Тогда вложение

$$
X_{1}^{i_{1}} X_{2}^{i_{2}} \ldots X_{m}^{i_{m}} \mapsto g_{i_{1}, \ldots, i_{m}},
$$

как нетрудно проверить, определяет изоморфизм.

В силу того, что любая абелева группа разлагается в прямое произведение циклических групп, получаем, что произвольная коммутативная групповая алгебра изоморфна некоторой фактор-алгебре алгебры многочленов от многих переменных, причем групповая алгебра циклической группы изоморфна фактор-алгебре алгебры многочленов от одной переменной. Сформулируем утверждение (см. [4]), которое будет использоваться для установления структуры групповой алгебры циклической группы.

Лемма 1. Если $f(X)$ и $g(X)$ - два взаимно простых многочлена от одной переменной над произвольным полем $k$, то

$$
k[X] /(f(X) g(X)) \cong k[X] /(f(X)) \times k[X] /(g(X)) .
$$

\section{2. Групповые алгебры минимального ранга}

Зафиксируем простое число $p$ и будем считать, что все рассматриваемые поля имеют характеристику $p$. Цель данного раздела - доказать необходимые и достаточные условия минимальности ранга групповой алгебры абелевой группы над полем простой характеристики. Как видно из теоремы Блезера, для того, чтобы алгебра была алгеброй минимального ранга, нужно, чтобы выполнялось условие (6) на структуру алгебры, а также условие на число элементов объемлющего поля. Если поле бесконечное, то условие на число элементов автоматически выполняется. Если же алгебра определена над конечным полем, то это условие является существенным.

Обозначим через $F_{q}$ конечное поле характеристики $p$ мощности $q$. Для натурального числа $n$, взаимно простого с $q$, определена мультипликативная степень числа $q$ по модулю $n$, то есть такое минимальное натуральное число $s_{n}$, что $q^{s_{n}}-1$ делится на $n$. Известно (см. [10]), что мультипликативная степень удовлетворяет следующим условиям:

(1) если $n$ и $m$ взаимно просты, то $s_{n m}=\operatorname{HOK}\left(s_{n}, s_{m}\right)$;

(2) если $n$ делит $m$, то $s_{n}$ делит $s_{m}$;

(3) $s_{n}$ делит $\varphi(n)$ для любого $n$, где $\varphi-$ функция Эйлера.

Разобьем множество $\mathscr{G}$ всех абелевых групп, на последовательность вложенных подмножеств. Пусть $G_{N}$ - произвольная абелева группа порядка $N$. Тогда группу $G_{N}$ можно однозначно представить в виде прямого произведения примарных циклических групп (см. [11])

$$
G_{N} \cong \mathbf{Z}_{p_{1}^{k_{1}}} \times \mathbf{Z}_{p_{2}^{k_{2}}} \times \ldots \times \mathbf{Z}_{p_{r}^{k r}}
$$


где $p_{1}, p_{2}, \ldots, p_{r}$ - простые числа. Будем говорить, что $G_{N} \in \mathscr{G}_{i}$, если не более чем $i$ из этих простых чисел совпадают с $p$. Очевидно, что $\mathscr{G}_{i} \subset \mathscr{G}_{i+1}, \mathscr{G}_{2}=\mathscr{G}_{0} \cup \mathscr{G}_{1} \cup \ldots \cup \mathscr{G}_{l} \cup \ldots$

Пусть $G_{N} \in \mathscr{G}_{1}$ - произвольная абелевая группа порядка $N$, принадлежащая множеству $\mathscr{G}_{1}$, и пусть $N=p^{k} n=p^{k} p_{1}^{k_{1}} p_{2}^{k_{2}} \ldots p_{r}^{k_{r}}$, где $p \nmid n, p_{1}, p_{2}, \ldots, p_{r}$ - попарно различные простые числа. Тогда

$$
G_{N} \cong \mathbf{Z}_{p^{k}} \times G_{n}
$$

где $G_{n}-$ произвольная абелевая группа порядка $n$. Пусть $G_{n}$ определяется следующим образом:

$$
\begin{gathered}
G_{n} \cong G_{p_{1}^{k_{1}}} \times G_{p_{2} k_{2}} \times \ldots \times G_{p_{r}^{k r}} . \\
G_{p_{i} k_{i}} \cong \mathbf{Z}_{p_{i} k_{i 1}} \times \mathbf{Z}_{p_{i} k_{i 2}} \times \ldots \times \mathbf{Z}_{p_{i}} \times k_{i r_{i}}, \\
0=k_{i 0}<k_{i 1} \leqslant k_{i 2} \leqslant \ldots \leqslant k_{i r_{i}}=l_{i}, \quad \sum_{j=1}^{r_{i}} k_{i j}=k_{i}, \quad i=1, \ldots, r .
\end{gathered}
$$

Пусть $m=p_{1}^{l_{1}} p_{2}^{l_{2}} \ldots p_{r}^{l_{r}}$. Заметим, что $m$ делит $n$ и что $m=n \Longleftrightarrow G_{n} \cong \mathbf{Z}_{n}$. Введем обозначение

$$
t= \begin{cases}2 p^{k} s_{m}-2, & \text { если } s_{m}>1, \\ 2 p^{k}-4, & \text { если } s_{m}=1\end{cases}
$$

где $s_{m}-$ мультипликативная степень числа $q$ по модулю $m$.

Теорема 3. Пусть $\mathbf{F}\left[G_{N}\right]$ - групповая алгебра абелевой группь порядка $N$ над произвольным полем простой характеристики. Алгебра $\mathbf{F}\left[G_{N}\right]$ является алгеброй минимального ранга тогда и только тогда, когда выполняются два условия:

(1) группа $G_{N}$ принадлежит множеству групп $\varphi_{1}$,

(2) если $\mathbf{F}$ - конечное поле, то число его элементов $q \geqslant t$.

Утверждение этой теоремы будет следовать из теорем 4, 5 и 6 этого раздела. При этом будем считать, что все обозначения, введенные выше, остаются справедливыми на протяжении всего раздела, за исключением подраздела 2.3.

\section{1. Групповые алгебры над конечным полем}

Пусть числа $u$ и $v$ таковы, что $1 \leqslant u \leqslant l_{i}, k_{i v}<u \leqslant k_{i v+1}$, то есть $v$ однозначно определяется по $u$. Пусть далее

$$
\sigma_{1}=1, \quad \sigma_{p_{i}^{u}}=p_{i}^{t_{i u}} \frac{p_{i}^{u\left(r_{i}-v\right)}-p_{i}^{(u-1)\left(r_{i}-v\right)}}{p_{i}^{u}-p_{i}^{u-1}}, \quad t_{i u}=\sum_{j=1}^{v} k_{i j} .
$$

Определим теперь числа $\sigma_{d}$, где $d-$ произвольный делитель числа $m$ :

$$
d=p_{1}^{u_{1}} p_{2}^{u_{2}} \ldots p_{r}^{u_{r}}, \quad 0 \leqslant u_{i} \leqslant l_{i}, \quad \sigma_{d}=\sigma_{p_{1}^{u_{1}}} \sigma_{p_{2}^{u_{2}}} \ldots \sigma_{p_{r}}^{u_{r}} .
$$


Введем обозначение

$$
\sigma_{F_{q}}=\sum_{d \mid m} \rho_{d} \sigma_{d}
$$

Пусть $\rho_{n}=\varphi(n) / s_{n}$. Многочлен $X^{n}-1$ разлагается на неприводимые множители над полем $F_{q}$ следующим образом (см. [10]):

$$
X^{n}-1=\prod_{d \mid n} \Phi_{d}(X)=\prod_{d \mid n} \prod_{j=1}^{\rho_{d}} f_{d j}(X),
$$

где $f_{d j}(X)$ - некоторый неприводимый многочлен степени $s_{d}$. Для любого натурального $d$ коэффициенты многочленов $\Phi_{d}$ принадлежат простому полю $F_{p}$.

Для любых неприводимых над полем $F_{q}$ многочленов $f(X)$ и $g(X)$ одинаковой степени $n$ алгебры $F_{q}[X] / f(X)$ и $F_{q}[X] / g(X)$ изоморфны и образуют конечное поле мощности $q^{n}$. Пользуясь этим, можно доказать, что для произвольного натурального $m$ алгебры $F_{q}[X] /(f(X))^{m}$ и $F_{q}[X] /(g(X))^{m}$ также изоморфны. Обозначим алгебру $F_{q}[X] /(f(X))^{m}$ через $F_{q}\langle n, m\rangle$.

Теорема 4. Пусть $F_{q}\left[G_{N}\right]$ - групповая алгебра абелевой группь порядка $N$ над конечным полем из q элементов, $G_{N} \in \mathscr{G}_{1}$. Тогда

$$
F_{q}\left[G_{N}\right] \cong \underset{d \mid m}{\chi}\left(F_{q}\left\langle s_{d}, p^{k}\right\rangle\right)^{\rho_{d} \sigma_{d}},
$$

и следующие условия эквивалентны:

(1) $F_{q}\left[G_{N}\right]$ - алгебра минимального ранга,

(2) $\operatorname{rk} F_{q}\left[G_{N}\right]=2 N-\sigma_{F_{q}}$,

(3) $q \geqslant t$.

Доказательство. Предположим, что верна формула (16) и докажем, что условия 1, 2 и 3 эквивалентны. Эквивалентность условий 1 и 2 означает, по определению алгебры минимального ранга, что число максимальных идеалов алгебры $F_{q}\left[G_{N}\right]$ равно $\sigma_{F_{q}}$. Известно (см. [5]), что

$$
t(A \times B)=t(A)+t(B),
$$

где $t(A)$ - число максимальных идеалов $A$. Таким образом, для доказательства эквивалентности условий 1 и 2 достаточно доказать, что каждая алгебра $F_{q}\left\langle s_{d}, p^{k}\right\rangle$ в формуле (16) имеет ровно один максимальный идеал.

Лемма 2. Для любых $n$ и $m$ алгебра $F_{q}[X] /(f(X))^{m}$ имеет ровно один максимальньий идеал, где $f(X)$ - произвольный неприводимый над $F_{q}$ многочлен степени $n$.

Доказательство. Пусть $I=(f(X))$ - идеал алгебры $F_{q}[X] /(f(X))^{m}$, порожденный многочленом $f(X)$, и пусть $g(X), h(X)$ - два произвольных элемента этой алгебры. Докажем, что если $g(X) \notin I$, то $h(X) \in(g(X))+I$. Заметим, что $g(X)$ и $h(X)$ можно представить в виде

$$
\begin{aligned}
& g(X)=\alpha_{m-1}(X)(f(X))^{m-1}+\alpha_{m-2}(X)(f(X))^{m-2}+\ldots+\alpha_{1}(X) f(X)+\alpha_{0}(X), \\
& h(X)=\beta_{m-1}(X)(f(X))^{m-1}+\beta_{m-2}(X)(f(X))^{m-2}+\ldots+\beta_{1}(X) f(X)+\beta_{0}(X),
\end{aligned}
$$


где

$$
\operatorname{deg} \alpha_{j}(X)<\operatorname{deg} f(X), \quad \operatorname{deg} \beta_{j}(X)<\operatorname{deg} f(X), \quad j=0,1, \ldots, m-1 .
$$

Так как $g(X) \notin I$, справедливо неравенство $\alpha_{0}(X) \neq 0$. Домножим $g(X)$ на элемент $\left(\alpha_{0}(X)\right)^{-1} \beta_{0}(X)$, где обратный элемент берется по модулю $f(X)$, и получим, что

$$
g(X)\left(\alpha_{0}(X)\right)^{-1} \beta_{0}(X)=\gamma_{m-1}(X)(f(X))^{m-1}+\ldots+\gamma_{1}(X) f(X)+\beta_{0}(X) .
$$

Значит,

$$
\begin{aligned}
h(X)=g(X)\left(\alpha_{0}(X)\right)^{-1} \beta_{0}(X) & +\left(\beta_{m-1}(X)-\gamma_{m-1}(X)\right)(f(X))^{m-1}+\ldots \\
& +\left(\beta_{m-1}(X)-\gamma_{m-1}(X)\right) f(X) \in(g(X))+I .
\end{aligned}
$$

Следовательно, $I$ - максимальный идеал.

Заметим, что $I^{m}=\{0\}$, поэтому $I-$ нильпотентный идеал. Радикал алгебры содержится в любом максимальном идеале, и любой нильпотентный идеал содержится в радикале, поэтому

$$
I=\operatorname{rad} F_{q}[X] /(f(X))^{m}
$$

Следовательно, $I$ - единственный максимальный идеал.

Докажем эквивалентность условий 1 и 3. Рассмотрим алгебру $F_{q}\left\langle s_{d}, p^{k}\right\rangle$, где $d$ делит $m$. Если $s_{d}>1$, то $F_{q}\left\langle s_{d}, p^{k}\right\rangle$ - локальная алгебра, и по теореме Блезера она является алгеброй минимального ранга тогда и только тогда, когда $q \geqslant 2 p^{k} s_{d}-2$. Если $s_{d}=1$, то, как видно из доказательства леммы $2, F_{q}\left\langle s_{d}, p^{k}\right\rangle$ - неразложимая сверхосновная алгебра, и $\operatorname{rad} F_{q}\left\langle s_{d}, p^{k}\right\rangle$ порождается одним элементом. Следовательно, также по теореме Блезера, алгебра $F_{q}\left\langle s_{d}, p^{k}\right\rangle$ является алгеброй минимального ранга тогда и только тогда, когда $q \geqslant 2\left(p^{k}-1\right)-2=2 p^{k}-4$. Так как $s_{d} \leqslant s_{m}$ для всех $d$, делящих $m$, получаем эквивалентность условий 1 и 3 .

Перейдем к доказательству формулы (16). Рассмотрим сначала алгебру циклической группы $F_{q}\left[\mathbf{Z}_{n}\right]$. В силу формулы (15) и леммы 1

$$
F_{q}\left[\mathbf{Z}_{n}\right] \cong F_{q}[X] /\left(X^{n}-1\right) \cong \underset{d \mid n}{X} F_{q}[X] / \Phi_{d}(X) \cong \underset{d \mid n}{X}\left(F_{q}\left\langle s_{d}, 1\right\rangle\right)^{\rho_{d}} .
$$

Рассмотрим теперь алгебру $F_{q}\left[G_{n}\right]$. Так как группа $G_{n}$ представляется в виде прямого произведения циклических групп, в силу (3) групповая алгебра $F_{q}\left[G_{n}\right]$ представляется в виде тензорного произведения алгебр циклических групп. Для того, чтобы воспользоваться теоремой Блезера, необходимо тензорное произведение алгебр представить в виде прямого произведения неразложимых алгебр.

Лемма 3. Если п и m-натуральные числа, взаимно простые с q, то

$$
F_{q}\left\langle s_{n}, 1\right\rangle \otimes F_{q}\left\langle s_{m}, 1\right\rangle \cong\left(F_{q}\left\langle\text { НОК }\left(s_{n}, s_{m}\right), 1\right\rangle\right)^{\text {НОД}\left(s_{n}, s_{m}\right)} .
$$

Доказательство. По определению алгебр $F_{q}\left\langle s_{n}, 1\right\rangle$ и $F_{q}\left\langle s_{m}, 1\right\rangle$, существуют неприводимые над $F_{q}$ многочлены $f(X)$ и $g(X)$ степеней $s_{n}$ и $s_{m}$, соответственно, такие, что $f(X)\left|\Phi_{n}(X), g(X)\right| \Phi_{m}(X)$ и

$$
F_{q}\left\langle s_{n}, 1\right\rangle \cong F_{q}[X] / f(X), \quad F_{q}\left\langle s_{m}, 1\right\rangle \cong F_{q}[X] / g(X) .
$$


Поэтому

$$
\begin{aligned}
F_{q}\left\langle s_{n}, 1\right\rangle \otimes F_{q}\left\langle s_{m}, 1\right\rangle & \cong F_{q}[X] / f(X) \otimes F_{q}[X] / g(X) \\
& \cong F_{q}[X, Y] /(f(X), g(Y))
\end{aligned}
$$

Обозначим через $F_{q}$ 的 поле $F_{q}[X] / f(X)$ мощности $q^{s_{n}}$. Произвольный элемент $a \in$ $F_{q}[X, Y] /(f(X), g(Y))$ представляется в виде

$$
a=f_{0}(X)+f_{1}(X) Y+\ldots+f_{s_{m}-1}(X) Y^{s_{m}-1},
$$

где $\operatorname{deg} f_{i}(X)<s_{n}$. Следовательно, элементы алгебры $F_{q}[X, Y] /(f(X), g(Y))$ можно рассматривать как элементы алгебры $F_{q}[Y] / g(Y)$, считая, что $f_{i}(X)-$ коэффициенты, принадлежащие $F_{q}$. Другими словами, можно так взаимно однозначно сопоставить элементы алгебр $F_{q}[X, Y] /(f(X), g(Y))$ и $F_{q^{s n}}[Y] / g(Y)$, что если перемножить произвольные два элемента алгебры $F_{q}[X, Y] /(f(X), g(Y))$ и перемножить соответствующие им элементы в алгебре $F_{q} s n[Y] / g(Y)$, то результаты этих умножений снова будут соответствовать друг другу.

Многочлен $g(Y)$ разлагается над $F_{q} s_{n}$ в произведение НОД $\left(s_{n}, s_{m}\right)$ неприводимых многочленов, степень каждого из которых равна $s_{m} / \mathrm{HОД}\left(s_{n}, s_{m}\right)$. Обозначим их $g_{1}(Y), \ldots, g_{l}(Y)$, где $l=$ НОД$\left(s_{n}, s_{m}\right)$. Тогда по лемме 1

$$
F_{q^{s n}}[Y] / g(Y) \cong F_{q^{s n}}[Y] / g_{1}(Y) \times \ldots \times F_{q^{s n}}[Y] / g_{l}(Y)
$$

Значит,

$$
F_{q}[X, Y] /(f(X), g(Y)) \cong F_{q}[X, Y] /\left(f(X), g_{1}(Y)\right) \times \ldots \times F_{q}[X, Y] /\left(f(X), g_{l}(Y)\right)
$$

Так как все конечные поля одинаковой мощности изоморфны друг другу, элементы поля $F_{q}[Y] / g_{i}(Y)$ можно рассматривать как элементы поля $F_{q}\left\langle\mathrm{HOK}\left(s_{n}, s_{m}\right), 1\right\rangle$. Следовательно,

$$
F_{q}[X, Y] /\left(f(X), g_{i}(Y)\right) \cong F_{q}\left\langle\operatorname{HOK}\left(s_{n}, s_{m}\right), 1\right\rangle, \quad i=1, \ldots, l .
$$

Лемма доказана.

Докажем, что

$$
F_{q}\left[G_{n}\right] \cong \underset{d \mid m}{X}\left(F_{q}[X] / \Phi_{d}\right)^{\sigma_{d}}
$$

Заметим, что из леммы 3 следуют два утверждения:

(a) если $n$ и $m$ взаимно просты, то

$$
F_{q}[X] / \Phi_{n} \otimes F_{q}[X] / \Phi_{m} \cong F_{q}[X] / \Phi_{n m},
$$

(б) если $k_{1} \geqslant k_{2}$, то

$$
F_{q}[X] / \Phi_{p_{i}^{k_{1}}} \otimes F_{q}[X] / \Phi_{p_{i}^{k_{2}}} \cong\left(F_{q}[X] / \Phi_{p_{i}^{k_{1}}}\right)^{\operatorname{dim} F_{q}[X] / \Phi_{p_{i} k_{2}}}, \quad i=1, \ldots, r .
$$


Рассмотрим алгебру $F_{q}\left[G_{p_{i}}\right]$ для $1 \leqslant i \leqslant r$. С учетом утверждения (б), используя формулы (17), (9), (3), (2), получаем, что $F_{q}\left[G_{p_{i}} k_{i}\right]$ изоморфна прямому произведению алгебр вида $F_{q}[X] / \Phi_{p_{i}^{u}}$, где $0 \leqslant u \leqslant l_{i}$. Обозначим через $\sigma_{p_{i}^{u}}$ кратность вхождения алгебры $F_{q}[X] / \Phi_{p_{i}^{u}}$ в указанное прямое произведение и докажем, что $\sigma_{p_{i}^{u}}$ определяется согласно формулам (12):

$$
\begin{aligned}
F_{q}\left[G_{p_{i}}\right] & \cong F_{q}\left[\mathbf{Z}_{p_{i}^{k_{i 1}}}\right] \otimes F_{q}\left[\mathbf{Z}_{p_{i}^{k_{i 2}}}\right] \otimes \ldots \otimes F_{q}\left[\mathbf{Z}_{p_{i}}\right] \\
F_{q}\left[\mathbf{Z}_{p_{i}}\right] & \cong F_{q}[X] / \Phi_{1} \times F_{q}[X] / \Phi_{p_{i}} \times \ldots \times F_{q}[X] / \Phi_{p_{i}} k_{i j}, \quad j=1, \ldots, r_{i} .
\end{aligned}
$$

Зафиксируем $u, 0 \leqslant u \leqslant l_{i}$, и определим $v$ из условия $k_{i v}<u \leqslant k_{i v+1}$. Заметим, что алгебра $F_{q}[X] / \Phi_{p_{i}^{u}}$ входит в разложение алгебры $F_{q}\left[\mathbf{Z}_{p_{i}} k_{i j}\right]$ при $j>v$. Поэтому, в силу утверждения (б), кратность вхождения алгебры $F_{q}[X] / \Phi_{p_{i}^{u}}$ в разложение алгебры $F_{q}\left[G_{p_{i}^{k_{i}}}\right]$ будет равна кратности ее вхождения в алгебру $F_{q}\left[\mathbf{Z}_{p_{i}^{k_{i 1}}}\right] \otimes \ldots \otimes F_{q}\left[\mathbf{Z}_{p_{i}^{k_{i v}}}\right] \otimes$ $F_{q}\left[\mathbf{Z}_{p_{i}^{u}}\right] \otimes \ldots \otimes F_{q}\left[\mathbf{Z}_{p_{i}^{u}}\right]$, где общее число сомножителей равно $r_{i}$. Суммарная размерность вхождения $F_{q}[X] / \Phi_{p_{i}^{u}}$ в разложение алгебры $F_{q}\left[\mathbf{Z}_{p_{i}^{u}}\right] \otimes \ldots \otimes F_{q}\left[\mathbf{Z}_{p_{i}^{u}}\right]$ равна суммарной размерности всех алгебр, входящих в это разложение, минус суммарная размерность алгебр, входящих в это разложение и отличных от алгебры $F_{q}[X] / \Phi_{p_{i}^{u}}$. Эта величина равна

$$
\begin{aligned}
\operatorname{dim}\left(F_{q}\left[\mathbf{Z}_{p_{i}^{u}}\right] \otimes \ldots \otimes F_{q}\left[\mathbf{Z}_{p_{i}^{u}}\right]-\operatorname{dim}\left(F_{q}\left[\mathbf{Z}_{p_{i}^{u-1}}\right] \otimes \ldots \otimes F_{q}\left[\mathbf{Z}_{p_{i}^{u-1}}\right]\right.\right. & \\
& =p_{i}^{u\left(r_{i}-v\right)}-p_{i}^{(u-1)\left(r_{i}-v\right)},
\end{aligned}
$$

где число сомножителей в каждой из круглых скобок равно $r_{i}-v$. Следовательно, кратность вхождения $F_{q}[X] / \Phi_{p_{i}^{u}}$ в указанное разложение равна

$$
\frac{p_{i}^{u\left(r_{i}-v\right)}-p_{i}^{(u-1)\left(r_{i}-v\right)}}{\operatorname{dim}\left(F_{q}[X] / \Phi_{p_{i}^{u}}\right)}=\frac{p_{i}^{u\left(r_{i}-v\right)}-p_{i}^{(u-1)\left(r_{i}-v\right)}}{p_{i}^{u}-p_{i}^{u-1}}
$$

Алгебра $F_{q}[X] / \Phi_{p_{i}^{u}}$ не входит в разложение алгебры $F_{q}\left[\mathbf{Z}_{p_{i}} k_{i 1}\right] \otimes \ldots \otimes F_{q}\left[\mathbf{Z}_{p_{i} k_{i v}}\right]$, поэтому окончательно получаем, что

$$
\begin{aligned}
\sigma_{p_{i}^{u}} & =\operatorname{dim}\left(F_{q}\left[\mathbf{Z}_{p_{i} k_{i 1}}\right] \otimes \ldots \otimes F_{q}\left[\mathbf{Z}_{p_{i} k_{i v}}\right]\right) \frac{p_{i}^{u\left(r_{i}-v\right)}-p_{i}^{(u-1)\left(r_{i}-v\right)}}{p_{i}^{u}-p_{i}^{u-1}} \\
& =p_{i}^{t_{i u}} \frac{p_{i}^{u\left(r_{i}-v\right)}-p_{i}^{(u-1)\left(r_{i}-v\right)}}{p_{i}^{u}-p_{i}^{u-1}} .
\end{aligned}
$$

В силу (8) и (3) для самой алгебры $F_{q}\left[G_{n}\right]$ получаем соотношение

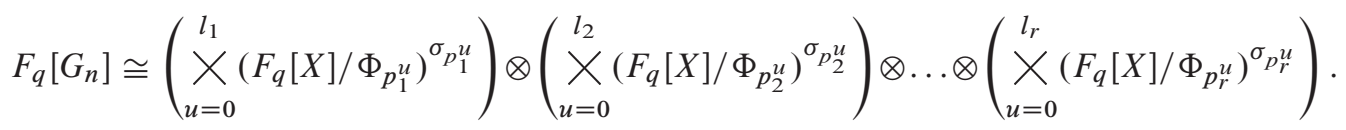

Применяя утверждение (а), приходим к формуле (19).

Докажем, что

$$
F_{q}\left[\mathbf{Z}_{p^{k}}\right] \otimes F_{q}\left\langle s_{n}, 1\right\rangle \cong F_{q}\left\langle s_{n}, p^{k}\right\rangle
$$


для любого $n$. Справедливы соотношения

$$
\begin{aligned}
F_{q}\left[\mathbf{Z}_{N}\right] & \cong F_{q}[X] /\left(X^{N}-1\right) \cong F_{q}[X] /\left(X^{n}-1\right)^{p^{k}} \\
& \cong \underset{d \mid n}{X}\left(F_{q}\left\langle s_{d}, p^{k}\right\rangle\right)^{\rho_{d}} .
\end{aligned}
$$

С другой стороны,

$$
\begin{aligned}
F_{q}\left[\mathbf{Z}_{N}\right] & \cong F_{q}\left[\mathbf{Z}_{p^{k}} \times \mathbf{Z}_{n}\right] \cong F_{q}\left[\mathbf{Z}_{p^{k}}\right] \otimes\left(\underset{d \mid n}{X}\left(F_{q}\left\langle s_{d}, 1\right\rangle\right)^{\rho_{d}}\right) \\
& \cong \underset{d \mid n}{X}\left(F_{q}\left[\mathbf{Z}_{p^{k}}\right] \otimes F_{q}\left\langle s_{d}, 1\right\rangle\right)^{\rho_{d}} .
\end{aligned}
$$

Так как все алгебры в формуле (20) является неразложимыми, каждая алгебра в формуле (21) изоморфна соответствующей алгебре в формуле (20). Таким образом,

$$
\begin{aligned}
F_{q}\left[G_{N}\right] & \cong F_{q}\left[\mathbf{Z}_{p^{k}}\right] \otimes F_{q}\left[G_{n}\right] \cong F_{q}\left[\mathbf{Z}_{p^{k}}\right] \otimes\left(\underset{d \mid m}{X}\left(F_{q}\left\langle s_{d}, 1\right\rangle\right)^{\rho_{d} \sigma_{d}}\right) \\
& \cong \underset{d \mid m}{X}\left(F_{q}\left\langle s_{d}, p^{k}\right\rangle\right)^{\rho_{d} \sigma_{d}} .
\end{aligned}
$$

Теорема 4 доказана.

Следствие 1. Ранг алгебры $F_{q}\left[G_{N}\right]$ равен ее размерности тогда и только тогда, когда $p \nmid N u m \mid q-1$.

\section{2. Групповые алгебры над бесконечным полем}

Обозначим через $\mathrm{F}$ произвольное бесконечное поле характеристики $p$. Для любого натурального $d$ коэффициенты многочлена $\Phi_{d}(X)$ принадлежат простому полю $F_{p}$, следовательно, так как $F_{p}$ - подполе $\mathrm{F}, \Phi_{d}(X)$ является многочленом над полем $\mathrm{F}$. Обозначим через $\rho_{d}$ число неприводимых над полем $\mathrm{F}$ многочленов, произведение которых равно многочлену $\Phi_{d}(X)$. Тогда

$$
\Phi_{d}(X)=f_{d 1}(X) f_{d 2}(X) \ldots f_{d \rho_{d}}(X),
$$

где многочлены $f_{d j}(X), j=1, \ldots, \rho_{d}$, неприводимы над полем F. Пусть

$$
\sigma_{\mathrm{F}}=\sum_{d \mid m} \rho_{d} \sigma_{d}
$$

Теорема 5. Пусть $\mathrm{F}\left[G_{N}\right]$ - групповая алгебра абелевой группы порядка $N$ над бесконечным полем характеристики $p, G_{N} \in G_{1}$. Тогда алгебра $\mathrm{F}\left[G_{N}\right]$ является алгеброй минимального ранга, $\operatorname{rkF}\left[G_{N}\right]=2 N-\sigma_{\mathrm{F}}, u$

$$
\mathrm{F}\left[G_{N}\right] \cong \underset{d \mid m}{\chi} \underset{j=1}{\rho_{d}}\left(\mathrm{~F}[X] /\left(f_{d j}(X)\right)^{p^{k}}\right)^{\sigma_{d}} .
$$


Доказательство. Из доказательства теоремы 4 видно, что

$$
F_{p}\left[G_{N}\right] \cong \underset{d \mid m}{\chi}\left(F_{p}[X] /\left(\Phi_{d}(X)\right)^{p^{k}}\right)^{\sigma_{d}} .
$$

Простое поле $F_{p}$ является подполем поля $\mathrm{F}$, поэтому

$$
\begin{aligned}
\mathrm{F}\left[G_{N}\right] & \cong \underset{d \mid m}{X}\left(\mathrm{~F}[X] /\left(\Phi_{d}(X)\right)^{p^{k}}\right)^{\sigma_{d}} \\
& \cong \underset{d \mid m}{X} \underset{j=1}{X}\left(\mathrm{~F}[X] /\left(f_{d j}(X)\right)^{\rho^{k}}\right)^{\sigma_{d}} .
\end{aligned}
$$

В силу бесконечности поля $\mathrm{F}$, по теореме Блезера алгебра $\mathrm{F}[X] /\left(f_{d j}(X)\right)^{p^{k}}$ является алгеброй минимального ранга и

$$
\operatorname{rkF}[X] /\left(f_{d j}(X)\right)^{p^{k}}=2 \operatorname{dim} \mathrm{F}[X] /\left(f_{d j}(X)\right)^{p^{k}}-1 .
$$

Следовательно, $\mathrm{F}\left[G_{N}\right]$ - алгебра минимального ранга и

$$
\operatorname{rkF}\left[G_{N}\right]=2 N-\sigma_{\mathrm{F}} .
$$

\section{3. Алгебры, не являющиеся алгебрами минимального ранга}

Для доказательства теоремы 3 осталось доказать, что условие $G_{N} \in \mathscr{G}_{1}$ является необходимым.

Теорема 6. Если $G_{N} \notin \mathscr{G}_{1}$, то $\mathbf{F}\left[G_{N}\right]$ не является алгеброй минимального ранга.

Доказательство. По условию теоремы, группа $G_{N}$ представляется в виде

$$
G_{N} \cong \mathbf{Z}_{p^{l_{1}}} \times \mathbf{Z}_{p^{l_{2}}} \times \ldots \times \mathbf{Z}_{p^{l_{m}}} \times G_{n}, \quad p \nmid n, \quad m \geqslant 2 .
$$

Введем обозначения

$$
M=p^{l_{1}} \ldots p^{l_{m}}, \quad G_{M} \cong \mathbf{Z}_{p^{l_{1}}} \times \ldots \times \mathbf{Z}_{p^{l_{m}}},
$$

и докажем, что алгебра $\mathbf{F}\left[G_{M}\right]$ не является алгеброй минимального ранга. Компоненты вектора алгебры $\mathbf{F}\left[G_{M}\right]$, равно как и элементы группы $G_{M}$, удобно нумеровать наборами из $m$ чисел $i_{1}, \ldots, i_{m}$, где $0 \leqslant i_{s}<p^{l_{s}}, s=1, \ldots, m$. При этом

$$
g_{i_{1}, \ldots, i_{m}} g_{j_{1}, \ldots, j_{m}}=g_{\left(i_{1}+j_{1}\right)} \quad\left(\operatorname { m o d } p ^ { l _ { 1 } ) , \ldots , ( i _ { m } + j _ { m } ) } \quad \left(\bmod p^{\left.l_{m}\right)} .\right.\right.
$$

Введем обозначения

$$
\begin{aligned}
h_{1} & =g_{1,0,0, \ldots, 0}-g_{0,0,0, \ldots, 0}, \\
h_{2} & =g_{0,1,0, \ldots, 0}-g_{0,0,0, \ldots, 0}, \\
& \ldots \\
h_{m} & =g_{0,0,0, \ldots, 1}-g_{0,0,0, \ldots, 0} .
\end{aligned}
$$

Рассмотрим идеал $I_{1}$, порожденный системой векторов $h_{1}, \ldots, h_{m}$, и докажем, что

$$
I_{1}=\operatorname{rad} \mathbf{F}\left[G_{M}\right]
$$


Легко проверить, что

$$
\left(h_{s}\right)^{p_{s}}=0, \quad\left(h_{s}\right)^{p^{l_{s}-1}} \neq 0, \quad s=1, \ldots, m .
$$

Следовательно, во-первых, $I_{1}-$ нильпотентный идеал, и, во-вторых, система векторов

$$
\left\{h_{1}^{i_{1}} h_{2}^{i_{2}} \ldots h_{m}^{i_{m}} \mid i_{1}+i_{2}+\ldots+i_{m} \geqslant 1,0 \leqslant i_{s}<p^{l_{s}}\right\} \subseteq I_{1}
$$

линейно независима. Значит,

$$
\operatorname{dim} I_{1}=p^{l_{1}} \ldots p^{l_{m}}-1=M-1
$$

и $I_{1}$ - максимальный идеал. Любой нильпотентный идеал содержится в радикале, а радикал содержится в любом максимальном идеале, поэтому $I_{1}=\operatorname{rad} \mathbf{F}\left[G_{M}\right]$.

Таким образом, $\mathbf{F}\left[G_{M}\right] / \operatorname{rad} \mathbf{F}\left[G_{M}\right] \cong \mathbf{F}$, то есть $\mathbf{F}\left[G_{M}\right]-$ сверхосновная алгебра. Воспользуемся критерием для сверхосновной алгебры из формулировки теоремы Блезера. Предположим, что существуют $w_{1}, \ldots, w_{t} \in \operatorname{rad} \mathbf{F}\left[G_{M}\right]$ такие, что $w_{i} w_{j}=0, i \neq j$, и

$$
\operatorname{rad} \mathbf{F}\left[G_{M}\right]=L R+w_{1} \mathbf{F}\left[G_{M}\right]+\ldots+w_{t} \mathbf{F}\left[G_{M}\right],
$$

где $L R-$ аннигилятор $\operatorname{rad} \mathbf{F}\left[G_{M}\right]$, базисом которого, очевидно, является единственный вектор $h_{1}^{p^{l_{1}-1}} \ldots h_{m}^{p^{l_{m}-1}}$. Обозначим $\alpha_{1}, \alpha_{2}$ коэффициенты перед векторами $h_{1}, h_{2}$, соответственно, в разложении вектора $w_{i}$ в базисе (24). Аналогично, через $\beta_{1}, \beta_{2}$ обозначим коэффициенты в разложении вектора $w_{j}$. Нетрудно проверить, что если $p>2$, то из равенства $w_{i} w_{j}=0$ следует, что

$$
\begin{aligned}
\alpha_{1} \beta_{2}+\alpha_{2} \beta_{1} & =0, \\
\alpha_{1} \beta_{1} & =0, \\
\alpha_{2} \beta_{2} & =0 .
\end{aligned}
$$

Если $p=2$, то из равенства $w_{i} w_{j}=0$ следует, что

$$
\alpha_{1} \beta_{2}+\alpha_{2} \beta_{1}=0
$$

над полем характеристики 2 это означает, что

$$
\alpha_{1} \beta_{2}=\alpha_{2} \beta_{1}
$$

В обоих случаях векторы $\alpha_{1} h_{1}+\alpha_{2} h_{2}$ и $\beta_{1} h_{1}+\beta_{2} h_{2}$ линейно зависимы.

Пусть $\pi$ есть оператор ортогонального проектирования в пространстве $\operatorname{rad} \mathbf{F}\left[G_{M}\right]$ на подпространство, порожденное векторами $h_{1}, h_{2}$, относительно подпространства, порожденного остальными векторами базиса (24). Как только что доказано, размерность пространства, порожденного векторами $\pi\left(w_{1}\right), \ldots, \pi\left(w_{m}\right)$, не превосходит 1. Применяя оператор $\pi$ к обеим частям (25), приходим к противоречию, так как слева получается пространство размерности 2, а справа - пространство размерности 1 .

Заметим, что алгебра $A \times B$ является алгеброй минимального ранга тогда и только тогда, когда обе алгебры $A$ и $B$ являются алгебрами минимального ранга. Это следует из теоремы Блезера в силу того, что все алгебры в (6) являются неразложимыми, и любая алгебра единственным образом разлагается в прямое произведение неразложимых алгебр. Следовательно, для завершения доказательства достаточно $\mathbf{F}\left[G_{N}\right]$ представить в виде $\mathbf{F}\left[G_{M}\right] \times A$, где $A-$ некоторая алгебра. 
Пусть

$$
G_{n} \cong \mathbf{Z}_{p_{1}^{k_{1}}} \times \mathbf{Z}_{p_{2}^{k_{2}}} \times \ldots \times \mathbf{Z}_{p_{r} k_{r}}
$$

где $p_{1}, \ldots, p_{r}$ - простые числа, не обязательно разные, но отличные от $p$. Тогда

$$
\begin{aligned}
\mathbf{F}\left[G_{N}\right] & \cong \mathbf{F}\left[G_{M}\right] \otimes \mathbf{F}\left[\mathbf{Z}_{p_{1}^{k_{1}}}\right] \otimes \ldots \otimes \mathbf{F}\left[\mathbf{Z}_{p_{r}} k_{r}\right] \\
& \cong \mathbf{F}\left[G_{M}\right] \otimes \mathbf{F}[X] /\left(X^{p_{1}^{k_{1}}}-1\right) \otimes \ldots \otimes \mathbf{F}[X] /\left(X^{p_{r}^{k_{r}}}-1\right)
\end{aligned}
$$

Так как $p_{i} \neq p, i=1, \ldots, r$, элемент 1 является простым корнем многочлена $X^{p_{i}^{k_{i}}}-1$ над полем $\mathbf{F}$, то есть многочлены $X-1$ и $\left(X^{p_{i}^{k_{i}}}-1\right) /(X-1)$ взаимно просты. Тогда

$$
\mathbf{F}[X] /\left(X^{p_{i}^{k_{i}}}-1\right) \cong \mathbf{F}[X] /(X-1) \times \mathbf{F}[X] /\left(\left(X^{p_{i}^{k_{i}}}-1\right) /(X-1)\right) \cong \mathbf{F} \times B_{i}
$$

Применяя (1) и (2), получаем, что

$$
\begin{aligned}
\mathbf{F}\left[G_{N}\right] & \cong \mathbf{F}\left[G_{M}\right] \otimes\left(\mathbf{F} \times B_{1}\right) \otimes \ldots \otimes\left(\mathbf{F} \times B_{r}\right) \\
& \cong \mathbf{F}\left[G_{M}\right] \otimes(\mathbf{F} \times B) \cong \mathbf{F}\left[G_{M}\right] \times A .
\end{aligned}
$$

Теоремы 6 и 3 доказаны.

\section{3. Верхняя и нижняя оценки билинейной сложности}

Из теоремы 4 следует, что по данной групповой алгебре $F_{q}\left[G_{N}\right]$, вычислив $t$ по формуле (11), можно определить, является ли эта алгебра алгеброй минимального ранга. Если является, то можно вычислить $\sigma_{F_{q}}$ и найти точное значение для rk $F_{q}\left[G_{N}\right]$. Если она не является алгеброй минимального ранга, то нижняя оценка $2 N-\sigma_{F_{q}}$ остается справедливой, тогда как о верхней оценке уже ничего сказать нельзя. Однако, используя представление (16), удается доказать, что билинейная сложность умножения в алгебре $F_{q}\left[G_{N}\right]$ растет линейно относительно ее размерности.

Для доказательства этого факта воспользуемся следующей известной теоремой, доказанной впервые в 1989 г. в [12].

Теорема 7. Существует константа $C$ такая, что для произвольного конечного поля $F_{q}$

$$
\operatorname{rk} F_{q}\langle n, 1\rangle \leqslant C n
$$

Теорема 8. Пусть $\mathbf{F}\left[G_{N}\right]$ - групповая алгебра абелевой группы порядка $N$ над произвольным полем характеристики $p, G_{N} \in \mathscr{G}_{l}$. Тогда существует константа $C_{l}$, зависящая только от множества $\mathscr{G}_{l}$, такая, что

$$
\operatorname{rk} \mathbf{F}\left[G_{N}\right] \leqslant C_{l} N
$$

Доказательство. По определению множества $\varphi_{l}$,

$$
\begin{aligned}
G_{N} \cong \mathbf{Z}_{p^{k_{1}}} \times \ldots \times \mathbf{Z}_{p^{k_{l}}} \times G_{n}, \quad G_{n} & \in \mathscr{G}_{0} \\
& \Longrightarrow \operatorname{rk} \mathbf{F}\left[G_{N}\right] \leqslant \operatorname{rk} \mathbf{F}\left[\mathbf{Z}_{p^{k_{1}}}\right] \ldots \operatorname{rk} \mathbf{F}\left[\mathbf{Z}_{p^{k_{l}}}\right] \operatorname{rk} \mathbf{F}\left[G_{n}\right]
\end{aligned}
$$


Пусть сначала $\mathbf{F}=F_{q}$ - конечное поле мощности $q$. В силу теоремы 4 ,

$$
F_{q}\left[G_{n}\right] \cong \underset{d \mid m}{\chi}\left(F_{q}\left\langle s_{d}, 1\right\rangle\right)^{\rho_{d} \sigma_{d}} \Longrightarrow \operatorname{rk} F_{q}\left[G_{n}\right] \leqslant \sum_{d \mid n} \rho_{d} \sigma_{d} \operatorname{rk} F_{q}\left\langle s_{d}, 1\right\rangle .
$$

Существует константа $C^{\prime}$ такая, что

$$
\text { rk } F_{q}\left\langle s_{d}, 1\right\rangle \leqslant C^{\prime} s_{d}
$$

для любого $s_{d}$, поэтому

$$
\operatorname{rk} F_{q}\left[G_{n}\right] \leqslant C^{\prime} n
$$

Докажем, что существует константа $C^{\prime \prime}$, для которой

$$
\operatorname{rk} F_{q}\left[\mathbf{Z}_{p^{k}}\right] \leqslant C^{\prime \prime} p^{k} \text {. }
$$

Рассмотрим алгебру $F_{q}[X] /\left(X^{p^{k}}-1\right)$, изоморфную алгебре $F_{q}\left[\mathbf{Z}_{p^{k}}\right]$. Умножение в этой алгебре состоит из двух частей:

(1) обычное умножение двух многочленов, степени которых меньше $p^{k}$;

(2) приведение полученного многочлена по модулю $X^{p^{k}}-1$.

Первую часть умножения можно выполнить в алгебре $F_{q}[X] / f(X)$, где $f(X)$ - неприводимый многочлен степени $2 p^{k}$. Так как степени исходных многочленов меньше $p^{k}$, умножение этих многочленов в алгебре $F_{q}[X] / f(X)$ совпадает с обычным умножением. Поэтому существует билинейный алгоритм, выполняющий первую часть умножения, сложность которого не превосходит $\operatorname{rk} F_{q}[X] / f(X)$. Из определения билинейного алгоритма нетрудно понять, что по данному билинейному алгоритму, вычисляющему произведение двух многочленов, можно построить билинейный алгоритм, вычисляющий это произведение по модулю $X^{p^{k}}-1$, той же длины, что и исходный алгоритм. Следовательно,

$$
\operatorname{rk} F_{q}\left[\mathbf{Z}_{p^{k}}\right] \leqslant \operatorname{rk} F_{q}[X] / f(X)=\operatorname{rk} F_{q}\left\langle 2 p^{k}, 1\right\rangle \leqslant 2 C^{\prime} p^{k}=C^{\prime \prime} p^{k} .
$$

Таким образом,

$$
\operatorname{rk} F_{q}\left[G_{N}\right] \leqslant C^{\prime \prime} p^{k_{1}} \ldots C^{\prime \prime} p^{k_{l}} C^{\prime} n=C_{l} N .
$$

Если $\mathbf{F}=\mathrm{F}-$ бесконечное поле, то

$$
\operatorname{rkF}\left[\mathbf{Z}_{p^{k_{1}}}\right] \ldots \operatorname{rkF}\left[\mathbf{Z}_{p^{k_{l}}}\right] \operatorname{rkF}\left[G_{n}\right] \leqslant 2 p^{k_{1}} \ldots 2 p^{k_{l}} 2 n=2^{l+1} N .
$$

Теорема 10 доказана.

Пусть $G_{1}, G_{2}, \ldots, G_{n}, \ldots$ - последовательность групп такая, что $G_{n} \in \mathscr{G}_{l_{n}} / \varphi_{l_{n}-1}$, $\left|G_{n}\right| \rightarrow \infty$ при $n \rightarrow \infty$, и последовательность $l_{n}$ ограничена сверху, то есть $l_{n} \leqslant l<\infty$. Из теоремы 5 следует, что над произвольным полем $\mathbf{F}$ характеристики $p$ верно, что

$$
\operatorname{rk} \mathbf{F}\left[G_{n}\right] \leqslant C \operatorname{dim} \mathbf{F}\left[G_{n}\right],
$$

где константа $C$ не зависит от $n$. При этом, остается открытым вопрос о том, как растет билинейная сложность $\operatorname{rk} \mathbf{F}\left[G_{n}\right]$ при $n \rightarrow \infty$, если последовательность $l_{n}$ неограничена, $\sup l_{n}=\infty$. Докажем некоторую нетривиальную нижнюю оценку для такой последовательности групповых алгебр над произвольным полем простой характеристики. Будем пользоваться следующей теоремой, доказанной в [13]. 
Теорема 9. Пусть $A-$ ассочииативная алгебра. Для всех $m, n>0$

$$
\text { rk } A \geqslant \operatorname{dim} A+\operatorname{dim}(\operatorname{rad} A)^{m}+\operatorname{dim}(\operatorname{rad} A)^{n}-\operatorname{dim}(\operatorname{rad} A)^{n+m-1} .
$$

В работе [13] была построена последовательность явно заданных алгебр $A_{n}$ с наилучшей среди известных нижней оценкой билинейной сложности, а именно, с оценкой $\left(3-\varepsilon_{n}\right) \operatorname{dim} A_{n}$, где $\varepsilon>0$ и $\varepsilon \rightarrow 0$ при $n \rightarrow \infty$. Оказывается, что последовательность алгебр с такой нижней оценкой можно выбрать и среди коммутативных групповых алгебр.

Теорема 10. Пусть $\mathbf{F}\left[G_{N}\right]-$ групповая алгебра группь

$$
G_{N} \cong \mathbf{Z}_{p} \times \mathbf{Z}_{p} \times \ldots \times \mathbf{Z}_{p}
$$

с числом сомножителей, равным $n$, над произвольным полем $\mathbf{F}$ характеристики $p, N=$ $p^{n}$. Тогда при $n \rightarrow \infty$

$$
\operatorname{rk} \mathbf{F}\left[G_{N}\right] \geqslant\left(3-\varepsilon_{n}\right) \operatorname{dim} \mathbf{F}\left[G_{N}\right],
$$

где $\varepsilon_{n}>0$ и $\varepsilon_{n} \rightarrow 0$ при $n \rightarrow \infty$.

Доказательство. Система векторов $\left\{h_{1}^{i_{1}} h_{2}^{i_{2}} \ldots h_{n}^{i_{n}} \mid i_{1}+i_{2}+\ldots+i_{n} \geqslant 1,0 \leqslant i_{s}<p\right\}$, как видно из доказательства теоремы 6 , образует базис идеала $I_{1}=\operatorname{rad} \mathbf{F}\left[G_{N}\right]$. Индукцией по $m$ легко показать, что система векторов $\left\{h_{1}^{i_{1}} h_{2}^{i_{2}} \ldots h_{n}^{i_{n}} \mid i_{1}+i_{2}+\ldots+i_{n} \geqslant m, 0 \leqslant i_{s}<p\right\}$ образует базис идеала $I_{m}=\left(\operatorname{rad} \mathbf{F}\left[G_{N}\right]\right)^{m}$. Следовательно,

$$
\operatorname{dim} I_{m+1}=p^{n}-a_{m},
$$

где $a_{m}$ - число элементов множества $\left\{\left(i_{1}, i_{2}, \ldots, i_{n}\right) \mid i_{1}+i_{2}+\ldots+i_{n} \leqslant m, 0 \leqslant i_{s}<p\right\}$.

В силу (27),

$$
\begin{aligned}
\operatorname{rk} \mathbf{F}\left[G_{N}\right] & \geqslant p^{n}+\left(p^{n}-a_{m}\right)+\left(p^{n}-a_{m}\right)-\left(p^{n}-a_{2 m}\right) \\
& =\left(3-\frac{p^{n}-a_{2 m}+2 a_{m}}{p^{n}}\right) p^{n} .
\end{aligned}
$$

Поэтому для завершения доказательства данной теоремы остается подобрать такое натуральное $m$, зависящее от $n$, что при $n \rightarrow \infty$

$$
\frac{a_{2 m}}{p^{n}} \rightarrow 1, \quad \frac{a_{m}}{p^{n}} \rightarrow 0 .
$$

Рассмотрим индексы $i_{1}, i_{2}, \ldots, i_{n}$ как независимые случайные величины, принимающие каждое целое значение из промежутка $[0, p-1]$ с вероятностью $1 / p$. Тогда случайная величина $\xi_{n}=i_{1}+i_{2}+\ldots+i_{n}$ принимает каждое целое значение $m$ из промежутка $[0, n(p-1)]$ с вероятностью $\left(a_{m}-a_{m-1}\right) / p^{n}$. Положим $m=\lfloor 2 n(p-1) / 3\rfloor$. Так как

$$
\mathbf{E} \xi_{n}=\frac{n(p-1)}{2}, \quad \mathbf{D} \xi_{n}=\frac{n\left(p^{2}-1\right)}{6},
$$

из неравенства Чебышева следует, что при $n \rightarrow \infty$

$$
\begin{array}{r}
\frac{a_{m}}{p^{n}}=\mathbf{P}\left(\xi_{n} \leqslant m\right) \leqslant \mathbf{P}\left(\left|\xi_{n}-\mathbf{E} \xi_{n}\right| \geqslant \mathbf{E} \xi_{n}-m\right) \leqslant \frac{\mathbf{D} \xi_{n}}{\left(\mathbf{E} \xi_{n}-m\right)^{2}} \rightarrow 0, \\
\frac{a_{2 m}}{p^{n}}=\mathbf{P}\left(\xi_{n} \leqslant 2 m\right) \geqslant \mathbf{P}\left(\left|\xi_{n}-\mathbf{E} \xi_{n}\right| \leqslant 2 m-\mathbf{E} \xi_{n}\right) \geqslant 1-\frac{\mathbf{D} \xi_{n}}{\left(2 m-\mathbf{E} \xi_{n}-1\right)^{2}} \rightarrow 1 .
\end{array}
$$




\section{Список литературы}

1. Cohn H., Umans C., A group-theoretic approach to fast matrix multiplication. In: Proc. 44th Annual IEEE Symp. FOCS. IEEE Computer Society, Los Alamitos, CA, 2003, pp. 438-449.

2. Cohn H., Kleinberg R. D., Szegedy B., Umans C., Group-theoretic algorithms for matrix multiplication. In: Proc. 46th Annual IEEE Symp. FOCS. IEEE Computer Society, Los Alamitos, CA, 2005, pp. 379-388.

3. Поспелов А. Д., Сложность умножения в ассоциативных алгебрах, Дисс. канд. физ.-мат. наук. МГУ, 2008.

4. Дрозд Ю. А., Кириченко В. В., Конечномерные алгебры. Вища школа, Киев, 1980.

5. Bürgisser P., Clausen M., Shokrollahi M., Algebraic complexity theory. Springer, Berlin, 1997.

6. Алексеев В. Б., Сложность умножения матриц. Обзор. Кибернетический сборник (1988) 25, 189-236.

7. Alder A., Strassen V., On the algorithmic complexity of associative algebras. Theoret. Comput. Sci. (1981) 15, 201-211.

8. Ван дер Варден Б. Л., Алгебра. Наука, Москва, 1979.

9. Bläser M., Algebras of minimal rank over arbitrary fields. Lect. Notes Comput. Sci. (2003) 2607, 403-414.

10. Берлекэмп Э., Алгебраическая теория кодирования. Мир, Москва, 1971.

11. Мельников О. В., Ремесленников В. Н., Романьков В. А., Скорняков Л. А., Шестаков И. П., Общая алгебра, 1. Наука, Москва, 1990.

12. Chudnovsky D., Chudnovsky G., Algebraic complexities and algebraic curves over finite fields. $J$. Complexity (1988) 4, 285-316.

13. Bläser M., Improvements of the Alder-Strassen bound: Algebras with nonzero radical. Lect. Notes Comput. Sci. (2001) 2076, 79-91. 